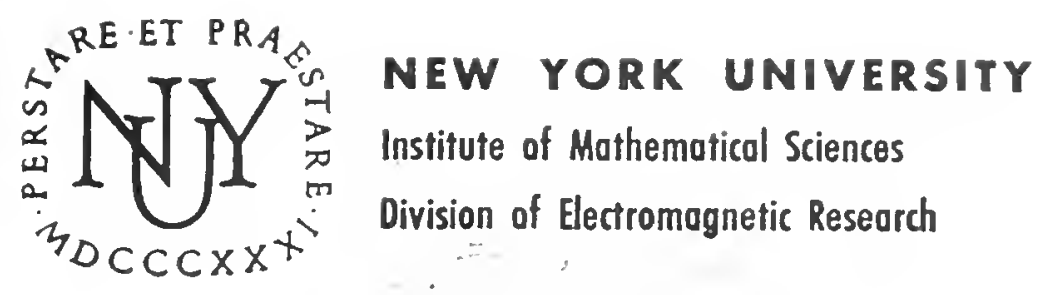

RESEARCH REPORT No. EM-72

\title{
The Behavior of Electromagnetic Fields at Edges
}

\author{
JOSEF MEIXNER
}

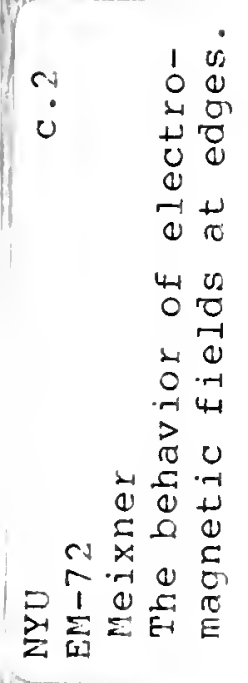

CONTRACT NO.AF-19(122)-42

DECEMBER 1954 
NEW YORK UNIVERSITY

Institute of Mathematical Sciences

Division of Electromagnetic Research

Research Report No. EM-72

THE BEHAVIOR OF ELECTROMAGNETIC FIELDS AT EDGES

Josef Meixner
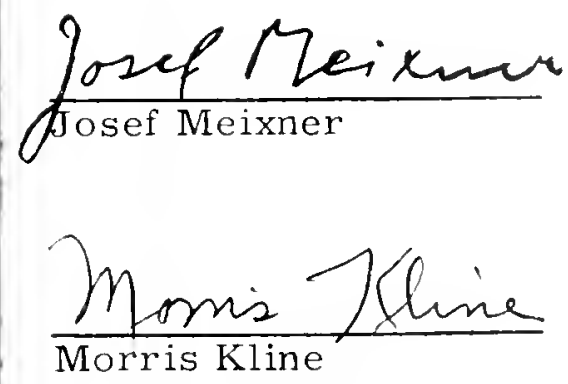

Project Director

The research reported in this document has been made possible through support and sponsorship extended by the Air Force Cambridge Research Center, under Contract No. AF-19(122)42. It is published for technical information only and does not necessarily represent recommendations or conclusions of the sponsoring agency. 


\section{ABSTRACT}

The behavior of an electromagnetic field in the neighborhood of the common edge of angular dielectric or conducting regions is determined from the condition that the energy density must be integrable over any finite domain (the so-called edge condition). Two cases are treated in detail, namely

1) A region consisting of a conducting wedge and two different dielectric wedges with a conmon edge.

2) A region consisting of two different dielectric wedges with a common edge.

It is also shown that near such edges electrostatic and magnetostatic fields will exhibit the same behavior as the electromagnetic field.

\section{Table of Contents}

\section{Introduction}

2. The behavior of fields in an angular domain 2

3. The case of figure 1 5

4. The case of figure 2 10

5. The electrostatic and magnetostatic cases 11 References 



\section{Introduction}

In the solution of diffraction problems it is found that at sharp edges of the diffracting obstacle the electromagnetic field vectors may become infinite. The order of this singularity is, however, subject to the so-called edge condition (Bouwkamp $[1]$, Meixner ${ }^{[2]}$ ), which states that the electromagnetic energy density must be integrable over any finite domain even if this domain contains singularities of the electromagnetic field; in other words the electromagnetic energy in any finite domain must be finite. In the case of a perfectly conducting surface with an edge one concludes from this condition that near the edge the singular components of the electric and magnetic field vectors are of the order $\rho^{-1 / 2}$, where $\rho$ is the distance from the edge, while the components of the electric and magnetic field strengths parallel to the edge are always finite.

In this paper we generalize the above result to find the behavior of the fie vectors near the edges of dielectric and perfectly conducting bodies. We restrict our selves to the neighborhoods of points for which there is a well-defined tangent along the edge. We may then consider the edge as locally straight. Hence it suffices to consider a space filled with wedges of homogeneous material with a common straight $f \mathrm{dg}$
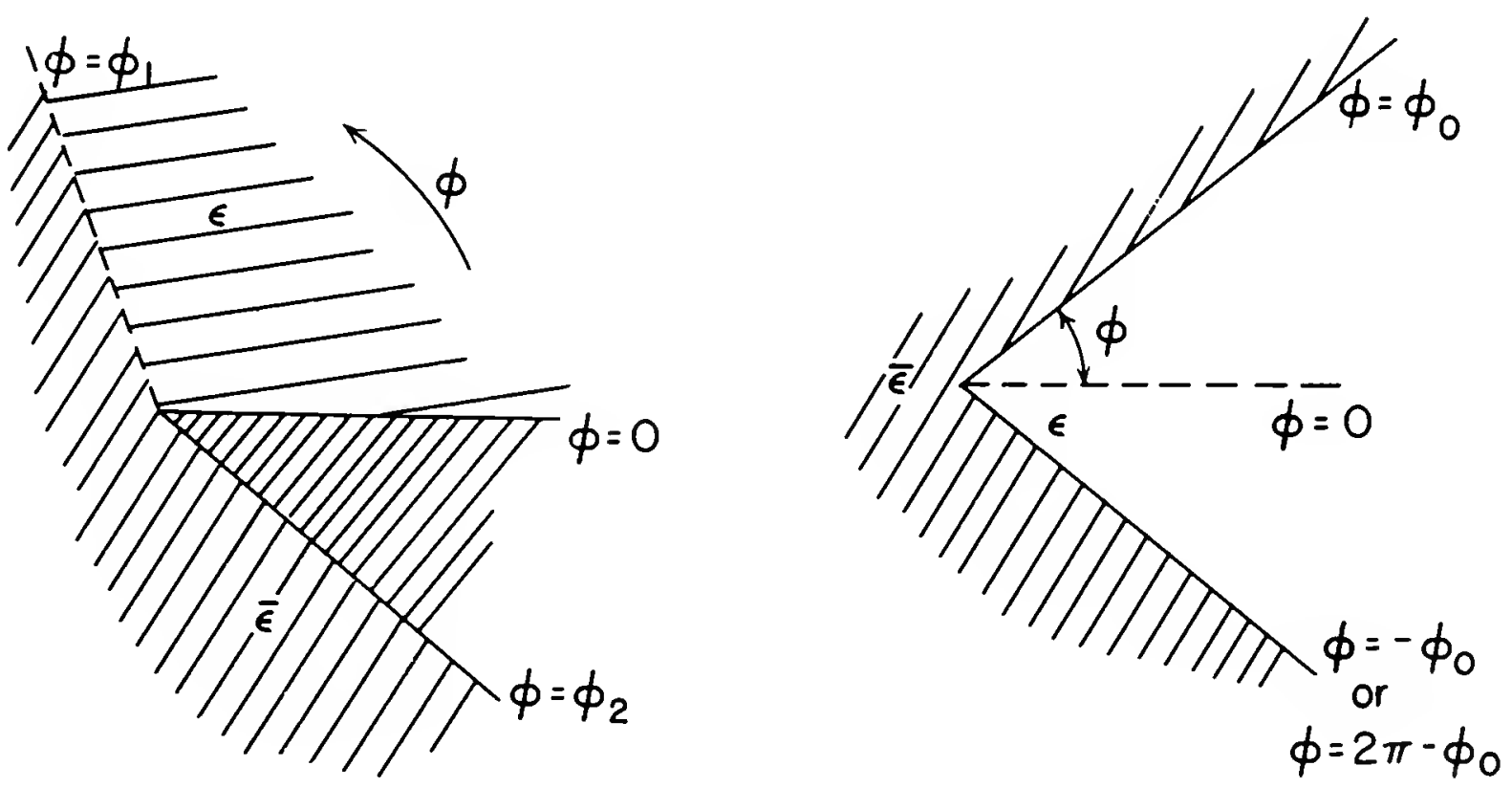

Fig. 1

Fig. 2 
We shall treat in detail two special cases, which are represented in cross section (perpendicular to the edge) in Figures 1 and 2.

Figure 1 represents a wedge $0 \leq \varnothing \leq \phi_{1}$ with dielectric constant $\varepsilon$, a wedge $\phi_{1} \leq \phi \leq \phi_{2}$ with dielectric constant $\bar{\varepsilon}$, and a wedge $\phi_{2} \leq \phi \leq 2 \pi$ of perfectly conducting material. Figure 2 represents a wedge $-\phi_{0} \leq \phi \leq \phi_{0}$ with dielectric constant $\varepsilon$ and magnetic permeability $\mu$ and a wedge $\phi_{0} \leq \phi \leq 2 n-\phi_{0}$ with dielectric constant $\bar{\varepsilon}$ and magnetic permeability $\bar{\mu}$.

\section{The behavior of fields in an angular domain}

We consider first Maxwell's equations in a homogeneous angular domain, for instance the wedge $0 \leq \phi \leq \phi_{1}$ in Figure 1 , and seek the behavior of the solutions of these equations near the edge. For periodis fields with circular frequency $\omega$ Maxwell's equations read

$$
i \omega \varepsilon \overline{\mathrm{E}}=\nabla \times \overline{\mathrm{H}}, \quad-i \omega \mu \mathrm{H}-\nabla \times \mathrm{E} \cdot
$$

Now let us introduce cylindrical coordinates $\rho, \phi, z$, with the $z$-axis along the edge and $\rho$ taken as the distance from the edge. Purther, let $E_{\rho}, E_{\phi}, E_{z}$ and $H_{\rho}, H_{\phi}, H_{z}$ be the components of the field vectors in cylindrical coordinates. Then Maxwell's cquations are

$$
\begin{aligned}
& \left\{\begin{aligned}
& i \omega \varepsilon E_{\rho}= \frac{1}{\rho} \frac{\partial z}{\partial \phi}-\frac{\partial H}{\partial z}, \\
& i \omega \varepsilon E_{\phi}=\frac{\partial H_{\rho}}{\partial z}-\frac{\partial z}{\partial \rho}, \\
& i \omega \varepsilon E_{z}=\frac{1}{\rho} \frac{\partial}{\partial \rho}\left(\rho H_{\phi}\right)-\frac{1}{\rho} \frac{\partial \rho}{\partial \phi} ;
\end{aligned}\right. \\
& \left\{\begin{array}{l}
-i \omega \mu H_{\rho}=\frac{1}{\rho} \frac{\partial E_{z}}{\partial \phi}-\frac{\partial E_{\phi}}{\partial z}, \\
-i \omega \mu H_{\phi}=\frac{\partial E}{\partial z}-\frac{\partial E}{\partial \rho}, \\
-i \omega \mu H_{z}=\frac{1}{\rho} \frac{\partial}{\partial \rho}\left(\rho E_{\varnothing}\right)-\frac{1}{\rho} \frac{\partial E_{\phi}}{\partial \rho} .
\end{array}\right.
\end{aligned}
$$


We now try to find solutions of these equations in the form

(3a)

$$
\begin{aligned}
& \left\{\begin{array}{l}
E_{\rho}=a_{0} \rho^{t-1}+a_{1} \rho^{t}+a_{2} \rho^{t+1}+\ldots, \\
E_{\varnothing}=b_{0} \rho^{t-1}+b_{1} \rho^{t}+b_{2} \rho^{t+1}+\cdots, \\
E_{z}=c_{0} \rho^{t-1}+c_{1} \rho^{t}+c_{2} \rho^{t+1}+\ldots ;
\end{array}\right. \\
& \left\{\begin{array}{l}
H_{\rho}=\alpha_{0} \rho^{t-1}+\alpha_{1} \rho^{t}+\alpha_{2} \rho^{t+1}+\ldots, \\
H_{\varnothing}=\beta_{0} \rho^{t-1}+\beta_{1} \rho^{t}+\beta_{2} \rho^{t+1}+\ldots, \\
H_{z}=\gamma_{0} \rho^{t-1}+\gamma_{1} \rho^{t}+\gamma_{2} \rho^{t+1}+\ldots,
\end{array}\right.
\end{aligned}
$$

where the coefficients depend only on $\phi$ and $z$. The admissible values of $t$ will be determined later from the boundary conditions at $\phi=\phi_{1}$ and $\phi=\phi_{2}$ in the case of Figure $I$ and at $\phi= \pm \phi_{0}$ in the case of igure 2. But we can already say that becanse of the edge conditions, $t$ must be positive.

Inserting the expansions ( $3 a$ ) and (3b) into Maxwell's equations (2a) and $(2 b)$ and comparing the coefficients of equal powers of $\rho$, we arrive at the following equations for the coefficients in $(3 a)$ and $(3 b)$ :

$$
c_{0}(t-1)=0, \quad \gamma_{0}(t-1)=0,
$$

$$
i \omega \varepsilon a_{0}=\frac{\partial \gamma_{1}}{\partial \phi}-\frac{\partial \beta_{0}}{\partial z} ;-i \omega \mu \alpha_{0}=\frac{\partial c_{1}}{\partial \varnothing}-\frac{\partial b_{0}}{\partial z} \text {, }
$$

$$
i \omega \varepsilon b_{0}=\frac{\partial a_{0}}{\partial z}-t \gamma_{1} ; \quad-i \omega \mu \beta_{0}=\frac{\partial a}{\partial z}-t c_{1} \text {, }
$$

$$
0=t \beta_{0}-\frac{\partial a}{\partial \phi} ; \quad 0=t b_{0}-\frac{\partial a_{0}}{\partial \phi}
$$

If we multiply the first equation of (4b) by $t$, differentiate the first equation of 
(4c) and ( $4 d$ ) with respect to $\varnothing$ and $z$ and add the resulting equations we get, assuning $\omega \not 0$

(4e)

$$
\frac{\partial b_{o}}{\partial \varnothing}=-t a{ }_{0}
$$

Combining this with the second equation of (4d) yields

$$
\frac{\partial^{2} a_{0}}{\partial \phi^{2}}+t^{2} a_{0}=0 \text {, }
$$

and by integration we obtain

$$
a_{0}=l_{1} \sin t \phi+\ell_{2} \cos t \phi
$$

By analogous procedure we get

$$
a_{0}=\lambda_{1} \sin t \phi+\lambda_{2} \cos t \phi,
$$

and then, using the fact that $t>0$, we readily obtain

$$
b_{0}=\ell_{1} \cos t \varnothing-\ell_{2} \sin t \varnothing \text {, }
$$

$$
\beta_{0}=\lambda_{1} \cos t \not \phi-\lambda_{2} \sin t \not \phi,
$$

$(6 e)$

$$
t c_{1}=\frac{\partial l_{1}}{\partial z} \sin t \phi+\frac{\partial l_{2}}{\partial z} \cos t \phi+i \omega \mu\left[\lambda_{1} \cos t \phi-\lambda_{2} \sin t \phi\right],
$$

$$
t r_{1}=\frac{\partial \lambda_{1}}{\partial z} \sin t \not+\frac{\partial \lambda_{2}}{\partial z} \cos t \not-i \omega \varepsilon\left[l_{1} \cos t \not-l_{2} \sin t \phi\right] \text {. }
$$

The coefficients $\ell_{1}, \ell_{2}, \lambda_{1}, \lambda_{2}$ are constants of integration with respect to $\phi$, but may depend on $z$.

If the field strenghts $\overline{\mathrm{E}}$ and $\overrightarrow{\mathrm{H}}$ are infinite at the edge, then $0<t<1$, and we get from (4a) $c_{0}=\gamma_{0}=0$. Thus it is proved that in any case the components of $\overline{\mathrm{E}}$ and $\overrightarrow{\mathrm{H}}$ parallel to the edge are finite. 
$-5-$

3. The case of Figure 1

The above considerations are quite general and are valid for any homogeneous angular domain. Now we apply our results to the case of Figure 1 . In the angular domain $0 \leq \varnothing \leq \phi_{1}$, the appropriate solution is given by $(6 a)-(6 f)$. In the angular domain $\phi_{1} \leq \phi \leq \phi_{2}$ the same solution is valid except that the coefficients $\bar{l}_{1}, \bar{l}_{2}$, $\bar{\lambda}_{1}, \bar{\lambda}_{2}$ and the parameters $\bar{\varepsilon}$ and $\bar{\mu}$ replace $l_{1}, l_{2}, \lambda_{1}, \lambda_{2}, \varepsilon_{1}$ and $\mu_{1}$ respectively. The boundary conditions are

$$
E_{p}=E_{z}=0 \text { for } \varnothing=0 \text { and } \phi=\phi_{2} \text {, }
$$

and we have the conditions of continuity

(8)

$$
E_{\rho}, E_{z}, H_{\rho}, H_{z} \text { continuous at } \varnothing=\phi_{I} \text {. }
$$

The conditions (7) lead to

(qa)

$$
\left\{\begin{array}{l}
l_{2}=0, \\
\lambda_{1}=0, \\
\bar{l}_{1} \sin t \phi_{2}+\bar{l}_{2} \cos t \phi_{2}=0, \\
\bar{\lambda}_{1} \cos t \phi_{2}-\bar{\lambda}_{2} \sin t \phi_{2}=0,
\end{array}\right.
$$

and the conditions (8) give

$(9 \mathrm{~b})$

$$
\left\{\begin{array}{l}
\left(l_{1}-\bar{l}_{1}\right) \sin t \phi_{1}+\left(l_{2}-\bar{l}_{2}\right) \cos t \phi_{1}=0, \\
\left(\lambda_{1}-\bar{\lambda}_{1}\right) \sin t \phi_{1}+\left(\lambda_{2}-\bar{\lambda}_{2}\right) \cos t \phi_{1}=0, \\
\left(\varepsilon l_{1}-\bar{\varepsilon} \bar{l}_{1}\right) \cos t \phi_{1}-\left(\varepsilon l_{2}-\bar{\varepsilon} \bar{l}_{2}\right) \sin t \phi_{1}=0, \\
\left(\mu \lambda_{1}-\bar{\mu} \bar{\lambda}_{1}\right) \cos t \phi_{1}-\left(\mu \lambda_{2}-\bar{\mu} \bar{\lambda}_{2}\right) \sin t \phi_{1}=0 .
\end{array}\right.
$$


Equations (9a) and (9b) separate into a set of homogeneous linear equations for $\ell_{1}, \ell_{2}, \bar{l}_{1}, \bar{l}_{2}$, and another such set for $\lambda_{1}, \lambda_{2}, \bar{\lambda}_{1}, \bar{\lambda}_{2}$. In order that not all of the $l_{1}, l_{2}, \bar{l}_{1}, \bar{l}_{2}$ vanish, one easily finds from the vanishing of the determinant of these equations that we must have

$$
\frac{\varepsilon-\bar{\varepsilon}}{\varepsilon+\bar{\varepsilon}}=\frac{\sin t \phi_{2}}{\sin t\left(2 \phi_{I}-\phi_{2}\right)} \text {. }
$$

Similarly, in order that not all the $\lambda_{1}, \lambda_{2}, \bar{\lambda}_{1}, \bar{\lambda}_{2}$ vanish we must have

$$
\frac{\bar{\mu}-\mu}{\bar{\mu}+\mu}=\frac{\sin t \phi_{2}}{\sin t\left(2 \phi_{1}-\phi_{2}\right)}
$$

For given values of $\bar{\varepsilon} / \varepsilon, \bar{\mu} / \mu, \phi_{1}$, and $\varnothing_{2}$, equations (10) and (11) dotermine values of $t$. For each solution $t$ of (10) there is a solution of $(9 a)$ and $(9 \mathrm{~b})$ with $\lambda_{1}=\lambda_{2}=\bar{\lambda}_{1}=\bar{\lambda}_{2}=0$, and for each solution $t$ of (II) there is a solution of $(9 a)$ and (9b) with $l_{1}=l_{2}=\bar{l}_{1}=\bar{l}_{2}=0$.

Figures 3 and 4 give representations of the curves $\phi_{1}$ versus $(\varepsilon-\bar{\varepsilon}) /(\varepsilon+\bar{\varepsilon})$ and $\phi_{1}$ versus $(\mu-\bar{\mu}) /(\mu+\bar{\mu})$ for a number of fixed values of t. Actually we represent

$$
\frac{\varepsilon-\bar{\varepsilon}}{\varepsilon+\bar{\varepsilon}}=\frac{\sin 2 \pi t^{*}}{\sin \left[2 \pi t^{*}\left(2 \phi^{*}-1\right)\right]}
$$

as a function of $\varnothing^{*}$ with

$$
\mathrm{t}^{*}=\frac{\phi_{2}}{2 \pi} \mathrm{t}, \quad \phi^{*}=\frac{\phi_{2}}{\phi_{2}},
$$

and correspondingly for $(\bar{\mu}-\mu) /(\bar{\mu}+\mu)$.

Interchanging $\varepsilon$ and $\bar{\varepsilon}$ or $\mu$ and $\bar{\mu}$ is equivalent to replacing $\emptyset_{1}$ by $\emptyset_{2}-\phi_{1}$. Therefore Figures 3 and 4 cover the whole range of values $0 \leq \phi_{1} \leq \phi_{2}$ if one takes the left and lower scale or the right and upper scale.

As is easily seen, there is an infinite set of solutions $t_{n}^{*}$ of (12) for prescribed $\bar{\varepsilon} / \varepsilon$ and $\phi^{*}$. Figures 3 and 4 correspond to the two snallest solutions. In the general case these solutions $t_{n}^{*}$ do not differ by integers, so that the be- 
havior of the electromagnetic field, which corresponds to a superposition of fields (3a), (3b) with all admissible values of $t$, may be very complicated. However, since we are interested here only in the case of electromagnetic fields which are infinite at the edge, we may confine ourselves to the solution of (10) and (11) in the interval $0<t<I$, i.e., to that part of Figures 3 and 4 for which $0<t^{*}<\frac{\phi_{2}}{2 \pi}$. of course the curves in Figures 3 and 4 are meaningful only for $-1<\frac{\varepsilon-\bar{\varepsilon}}{\varepsilon+\bar{\varepsilon}}<1$ or $-1<\frac{\mu-\bar{\mu}}{\mu+\bar{\mu}}<1$ (we leave out of consideration media with jielectric or magnetic losses, that is, media for which the dielectric constant and the magnetic permeability could assume complex or even negative values). Now from (12) it follows immediately that these conditions are not satisfied if $0<t^{* *} \leq \frac{1}{4}$ for arbitrary values of $\phi^{* k}\left(0<\phi^{* *}<1\right)$; hence we have the further restriction $t^{*}>\frac{1}{4^{*}}$. Thus, no infinite fields occur if $\frac{\phi_{2}}{2 \pi} \leq \frac{1}{4}$ or $\phi_{2} \leq \frac{x}{2}$.

Some special cases are worth mentioning.

1. If $\varepsilon=\bar{\varepsilon}$, then a solution with $t=\pi / \phi_{2}$ exists. This result has been given for $\phi_{2}=2 \pi$ (edge of a perfectly conducting half-plane) by lieixner [2], and for arbitrary values of $\phi_{2}$ by Maue ${ }^{[3]}$. For $\phi_{2}<\pi$, we have $t>1$, and then all field quantities remain finite.

If $\bar{\mu} \neq \mu$, then in general there can exist another solution for which $t \neq \pi / \phi_{2}$

2. If $\mu=\bar{\mu}$, the same considerations apply, with $\varepsilon$ and $\mu$ interchanged.

3. If $\phi_{1}=0$ or $\phi_{1}=\phi_{2}$, there is only one dielectric medium and both equations (10) and (17) are satisfied identically if we choose $t=\pi / \phi_{2}$, for arbitrary $\overline{8} / \varepsilon, \bar{\mu} / \mu$; and no other solution $t>1$ exists.

4. If $\phi_{1}=\phi_{2} / 2$ (we call this the symmetric case), then equations (10) or (11) again can be satisfied, apart from finite solutions of Maxwell's equations, only if $t=\pi / \phi_{2}$. In other words, in the symmetric case the exponent $t$ is 
independent of the electrical and magnetic properties of the two media. This is the situation which occlirs for example in the case of diffraction by a slit in a conductor between two different dielectric media [4].

Thus we conclude that the lines $(\varepsilon-\bar{\varepsilon}) /(\varepsilon+\bar{\varepsilon})=0($ and $(\bar{\mu}-\mu) /(\bar{\mu}+\mu)-0)$, $\phi_{1}=0, \phi_{1}=\phi_{2} / 2$ and $\phi_{1}=\phi_{2}$ in Figure 3 belong to the same value $t^{*}=\frac{1}{2}$ or $t=\pi / \phi_{2}$.

4. The case of Fingure 2

In the case of Figure 2 we take the solution (6a) - (6f) for the angular domain $-\phi_{0} \leq \varnothing<\phi_{0}$ and the corresponding solution with the coefficients $\bar{l}_{1}, \bar{l}_{2}$, $\bar{\lambda}_{1}, \bar{\lambda}_{2}, \bar{\varepsilon}, \bar{\mu}$ for the angular domain $\phi_{0} \leq \phi \leq 2 \pi-\phi_{0}$. The continuity of $E_{\rho}, E_{z}, H_{\rho}$, $\mathrm{H}_{\mathrm{z}}$ for $\phi=-\phi_{0}$ or $2 \pi-\phi_{0}$ and for $\phi=\phi_{0}$ leads to the equations

$$
\begin{aligned}
-l_{1} \sin t \phi_{0}+l_{2} \cos t \phi_{0} & =\bar{l}_{1} \sin t\left(2 \pi-\phi_{0}\right)+\bar{l}_{2} \cos t\left(2 \pi-\phi_{0}\right), \\
\varepsilon & {\left[l_{1} \cos t \phi_{0}+\ell_{2} \sin t \phi_{0}\right]=\bar{\varepsilon}\left[\bar{l}_{1} \cos t\left(2 \pi-\phi_{0}\right)-\bar{l}_{2} \sin t\left(2 \pi-\phi_{0}\right)\right], } \\
l_{1} \sin t \phi_{0}+l_{2} \cos t \phi_{0} & =\bar{l}_{1} \sin t \phi_{0}+\bar{l}_{2} \cos t \phi_{0}, \\
\varepsilon\left[l_{1} \cos t \phi_{0}-l_{2} \sin t \phi_{0}\right] & =\bar{\varepsilon}\left[\bar{l}_{1} \cos t \phi_{0}-\bar{l}_{2} \sin t \phi_{0}\right] .
\end{aligned}
$$

The same equations hold with $\lambda_{1}, \lambda_{2}, \bar{\lambda}_{1}, \bar{\lambda}_{2}, \mu, \bar{\mu}$ in place of $l_{1}, l_{2}, \bar{l}_{1}$, $\bar{l}_{2}, \varepsilon, \bar{\varepsilon}_{\text {. }}$ One finds that $t$ must satisfy one of the following four equations:

$$
\begin{aligned}
& \frac{\bar{\varepsilon}-\varepsilon}{\bar{\varepsilon}+\varepsilon}=\frac{\sin t \pi}{\sin t\left(2 \phi_{0}-\pi\right)} \text { with } \ell_{1}=0, \lambda_{1}=\lambda_{2}=\pi_{1}=\lambda_{2}=0, \\
& \frac{\varepsilon-\bar{\varepsilon}}{\varepsilon+\bar{\varepsilon}}=\frac{\sin t \pi}{\sin t\left(2 \phi_{0}-\pi\right)} \text { with } l_{2}=0, \lambda_{1}=\lambda_{2}=\bar{\lambda}_{1}=\bar{\lambda}_{2}=0, \\
& \frac{\bar{\mu}-\mu}{\bar{\mu}+\mu}=\frac{\sin t \pi}{\sin t\left(2 \phi_{0}-\pi\right)} \text { with } \lambda_{1}=0, \ell_{1}=l_{2}=\bar{l}_{1}=\bar{l}_{2}=0, \\
& \frac{\mu-\bar{\mu}}{\mu+\bar{\mu}}=\frac{\sin t \pi}{\sin t\left(2 \phi_{0}-\pi\right)} \text { with } \lambda_{2}=0, l_{1}=l_{2}=\bar{l}_{1}=\bar{l}_{2}=0,
\end{aligned}
$$


where the vanishing coefficients indicate the types of the solutions. One sees immedlately from these equations, comparing them with (10) and (11), that the admissible values of $t$ can again be taken from Figure 3 , if we choose there $\phi_{2}=\pi, \phi_{1}=\phi_{0}, t^{*}=\frac{t}{2}$ and take as the vertical axis either $(\varepsilon-\bar{\varepsilon}) /(\varepsilon+\bar{\varepsilon})$ and $(\bar{\mu}-\mu) /(\bar{\mu}+\mu)$ or $(\bar{\varepsilon}-\epsilon) /(\bar{\varepsilon}+\varepsilon)$ and $(\mu-\bar{\mu}) /(\mu+\bar{\mu})$. For given values of $\bar{\varepsilon} / \epsilon$ or $\bar{\mu} / \mu$ and $\phi_{0}$ there exist two solutions of Maxwell's equations. These solutions generally have different values of $t$, but one value of $t$ is always larger than unity and so one solution must be finite. It might be worth noting that in the case where there is no wedge, that is, when $\varepsilon=\bar{\varepsilon}, \mu=\bar{\mu}$, the admissible values of $t$ are given by $\sin t \pi=0$, and since the values $t=-1,-2, \ldots$ are excluded by the edge condition, the electromagnetic field is regular, as it must be.

\section{The electrostatic and magnetostatic cases.}

The electrostatic and the magnetostatic cases must be treated separately, since in the foregoing considerations we have assumed $\omega \neq 0$. For $\omega=0$, equations (5) cannot be derived from (4b) to (4d); in this case Maxwell's equations $\nabla \cdot \mathrm{E}-0$ and $\nabla \cdot \mathrm{H}=\mathrm{O}$ (in homogeneous and charge-free domains) no longer follow from (1), but are independent additional equations. Taking them into account we arrive again at the results $(6 a)$ to $(6 f)$.

There is still another difference in the argument. The conditions of continulty along a surface separating two different dielectric media give a factor

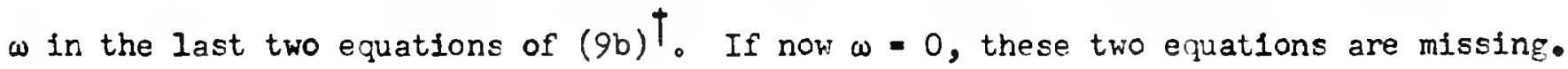
However, they do appear if we apply the continulty condition for the normal component of the dielectric displacement $\varepsilon \overline{\mathbf{E}}$ and the magnetic induction $\mu \bar{H}$, provided there are no surface charges. Thus all our results are valid also in the electrostatic or magnetostatic case, if the surface separating differentmaterials do not carry surface charges. The electrostatic case was treated by Karp [5] inlependently, and the same results were obtained. $t$ This factor has been omitted since we assumed $\omega \neq 0$. 
References

[1] C. Bouwkamp, A Note on Singularities Occurring at Sharp Edges in Electromagnetic Diffraction Theory, Physica, 12, 467, (1946).

[2] J. Meixner, Tie Kantenbedingung in der Theorie der Beugung electromagnetischer wellen an vollkommen leitenden ebenen Schirmen, Ann. Phys. (6) 6,1-9 (1949).

[3] A. W. Maue, Zur Formulierung eines allgemeinen Beugungs-problems durch. eine Integralgleichung, Z. Phys. 126, 601 (1949).

[4] J. Meixner, Diffraction of Electromagnetic Waves by a Slit in a Conducting Plane between Different Media, New York University, Institute of Mathematical Sciences, Division of Electromagnetic Research, Research Report No. EM-68, Oct., 1954.

[5] S. N. Karp, The Effect of Discontimities of Dielectric Constant on Flelds near Singularities of Conductors, New York University, Institute of Mathematical Sciences, Division of Electramagnetic Research, Research Report No. EM-PI, Dec., 1954. 


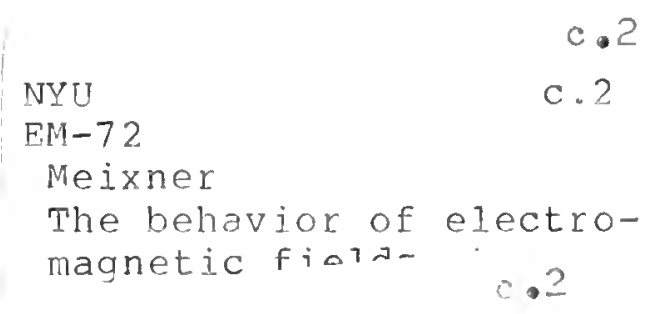

NYI

$E M-72$

Meixner
The behavior of electromagnetic fields at edges

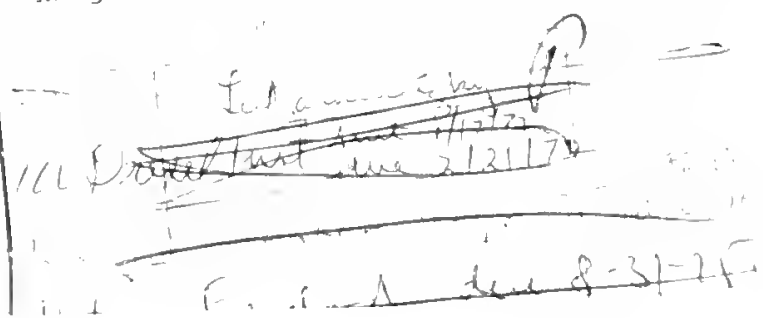

N. Y. U. Courant Institute of Mathematical Sciences

4 Washington Place

New York 3, N. Y. 

3

.

H.

i)

(1)

4

4

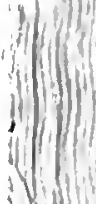

-

.

i)

1

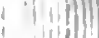

: II
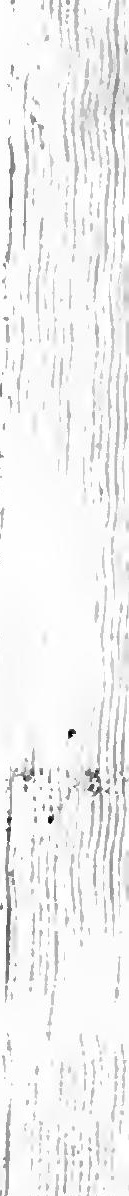